\title{
Efficiency Bounds for Sequential Resource Allocation Auctions
}

\author{
Junjik Bae*, Eyal Beigman ${ }^{\dagger}$, Randall Berry*, Michael L. Honig*, and Rakesh Vohra ${ }^{\dagger}$ \\ *EECS Department \\ Northwestern University, Evanston, IL 60202 \\ junjik@northwestern.edu, \{rberry, mh\}@ece.northwestern.edu \\ $\dagger$ CMS-EMS, Kellogg School of Management \\ Northwestern University, Evanston, IL 60208 \\ \{e-beigman,r-vohra\}@northwestern.edu
}

\begin{abstract}
Market-based mechanisms such as auctions have been widely considered for various network resource allocation problems. We consider such a mechanism motivated by dynamic spectrum sharing applications. In this model multiple homogeneous units of a given resource are to be allocated to two agents. We study a sequential second price auction for allocating these resource units. It is well known that such auctions can have inefficient equilibria. For the case of two bidders, we show that the value of the allocation obtained in the unique subgame perfect equilibrium is at least $1-e^{-1}$ of the value of the efficient allocation. Furthermore, we show that this bound is asymptotically tight as the number of goods increases.
\end{abstract}

\section{INTRODUCTION}

Market-based mechanisms such as auctions provide a natural approach for allocating any constrained resource among various agents. Such approaches have been studied for a variety of different resource allocation problems that arise in communication systems such as allocating capacity on a communication link, e.g. [1], [2] or dynamically allocating wireless spectrum usage, e.g. [3], [4]. Our work here is motivated by the second application. Specifically, we are motivated by the case where a spectrum manager allocates spectrum to users on a secondary market (These types of secondary markets for spectrum allocation are currently being considered by the FCC [5], [6]). For example, spectrum sharing could be achieved by allowing each user to have exclusive use of a portion of the available bandwidth. Though motivated by this application, the problem we consider applies in a much more general setting.

We consider the case where the available resource is divided into $n$ homogeneous goods and each good is to be allocated to one of two competing agents. The agents are endowed with quasi-linear utilities that satisfy diminishing marginal valuations. The mechanism we consider is a sequential second price auction. In this mechanism, the goods are allocated sequentially in a sequence of $n$ rounds. In each round, the two agents bid for the current unit. The unit is allocated to the highest bidder at the price of the second highest bid. This auction is repeated until all $n$ units are allocated.

For example, in a spectrum sharing application each good could represent a fixed unit of bandwidth. In such a setting,

This research was supported in part by NSF under grant CNS-0519935. there are a number of reasons why one may want to use a sequential auction. For example, sequential auctions impose much less of a computational burden on the manager than in many other auctions. Sequential auctions also accommodate scenarios where agents can enter and leave the market at arbitrary times, and allow the manager to auction off pieces of the spectrum incrementally.

The study of the sequential auctions is not new; see for example [7], [8], [9], and [10]. The rise of eBay has renewed interest in such auctions as stylized models of the eBay market place (see for example [11], [12]). In these papers valuations are assumed to be private but bidders have unit demand. In our case an agent is interested in consuming more than one unit. This makes the private information of agents multidimensional. To allow for tractability, we will assume that valuations are common knowledge.

It is well known that for $n=1$ the second price auction is efficient, namely, the unit is allocated to the agent who has the highest valuation. However, for $n>1$, it might have inefficient equilibria [10]. In other words, considering the sequential second price auction as an extensive form game, the allocation of goods obtained in a subgame perfect equilibria of this game may not be the allocation which maximizes the total utility of the agents. In the spirit of [2], [13] we attempt to bound this loss in efficiency. Our main results show that the worst-case efficiency is always at least $1-e^{-1}$ of the value of the efficient allocation. This bound is asymptotically tight, as $n \rightarrow \infty$. In addition, our examples illustrate that the worstcase efficiency occurs when the smallest marginal valuation of one agent is larger than the largest marginal valuation of the other agent.

\section{THE MODEL}

We consider the case of 2 agents and $n$ homogeneous goods. In this setting, the sequential second price auction can be modeled as an extensive form game with a balanced binary game tree. Each decision node on the game tree designates an intermediate state of the world, where a certain quantity of units are allocated to agent 1 and 2. Since all of the $n$ goods are homogeneous, all the decision nodes with the same allocation can be unified and the game tree can be replaced with a directed graph $G=(V, E)$, where $V=\left\{\left(s_{k}, t_{k}\right) \in\right.$ 
$[0, \ldots, n] \times[0, \ldots, n] \mid s_{k}+t_{k}=k$ for $\left.k=0,1, \ldots, n\right\}$ (see Fig. 1). A node $\left(s_{k}, t_{k}\right) \in V$ represents the outcome of the $k$-th round of the auction, in which agent 1 has been allocated $s_{k}$ units and agent 2 has been allocated $t_{k}$ units. The directed edge from a certain node indicates who wins the current unit in the sequential auction. If $s_{k}+t_{k}=k<n$, each node $\left(s_{k}, t_{k}\right)$ branches to either node $\left(s_{k}+1, t_{k}\right)$ or $\left(s_{k}, t_{k}+1\right)$ depending on the bids of both agents. The auction begins at the root node $\left(s_{0}, t_{0}\right)=(0,0)$.

Let $u_{l}^{i}$ denote the marginal value of agent $i$ for the $l$-th good she receives. We assume both agents have decreasing marginal values ${ }^{1}$, i.e. $u_{1}^{i} \geq \ldots \geq u_{n}^{i}$. Agent 1 's utility for allocation $\left(s_{n}, t_{n}\right)$ is therefore $\sum_{j=1}^{s_{n}} u_{j}^{1}$, and that of agent 2 is $\sum_{j=1}^{t_{n}} u_{j}^{2}$. Let $H$ be the set of observable bidding histories. A strategy $\sigma_{i}: V \times H \rightarrow \mathbb{R}^{+}$is a function mapping states of the allocation and observable histories to bids. The strategy set of an agent is the set of all such functions. The outcome path of a pair of strategies $\left\{\sigma_{1}\right\}$ and $\left\{\sigma_{2}\right\}$ is a sequence of vertices $\delta=\left\{\left(s_{0}, t_{0}\right), \ldots,\left(s_{n}, t_{n}\right)\right\}$ such that $s_{k+1}=s_{k}+1$ and $t_{k+1}=t_{k}$ if and only if

$$
\sigma_{1}\left(\left(s_{k}, t_{k}\right), \Gamma_{k}\right)>\sigma_{2}\left(\left(s_{k}, t_{k}\right), \Gamma_{k}\right),
$$

and $s_{k+1}=s_{k}$ and $t_{k+1}=t_{k}+1$ if and only if

$$
\sigma_{1}\left(\left(s_{k}, t_{k}\right), \Gamma_{k}\right) \leq \sigma_{2}\left(\left(s_{k}, t_{k}\right), \Gamma_{k}\right),
$$

where $\Gamma_{k}$ is the bidding history on the path for the first $k$ units. $^{2}$

For a given pair of strategies, $\left(\sigma_{1}, \sigma_{2}\right)$, the payment of agent 1 at $\left(s_{k}, t_{k}\right)$ is

$$
p_{1}\left(s_{k}, t_{k}\right)=\sigma_{2}\left(\left(s_{k}, t_{k}\right), \Gamma_{k}\right),
$$

if $\sigma_{1}\left(\left(s_{k}, t_{k}\right), \Gamma_{k}\right)>\sigma_{2}\left(\left(s_{k}, t_{k}\right), \Gamma_{k}\right)$ and $p_{1}\left(s_{k}, t_{k}\right)=0$ otherwise. The total payment of agent $i$ along the path $\Gamma$ is $P_{i}(\Gamma)=\sum_{k=0}^{n-1} p_{i}\left(s_{k}, t_{k}\right)$.

\section{A. Bidding Strategies}

There are two basic types of bidding strategies that are of special interest. A bidding strategy is myopic if it maximizes payoff over intermediate outcomes. An extreme case of myopic bidding is bidding the marginal value of the current unit on each round, which we call greedy bidding. Formally, $\sigma_{1}\left(\left(s_{k}, t_{k}\right), \Gamma_{k}\right)=u_{s_{k}+1}^{1}$ and $\sigma_{2}\left(\left(s_{k}, t_{k}\right), \Gamma_{k}\right)=u_{t_{k}+1}^{2}$ are the greedy strategies. As we will see in Section II-B, greedy bidding is no longer a dominant strategy when there is more than one unit. This does not imply that this strategy cannot be rationalized. In contrast it can be associated with some sort of maximizing behavior depending on the information structure of the extensive form game. In the case where the agents are uninformed about the number of units on the market or the valuation of the other agents, it may be rational to bid the marginal value every round under the belief that the current

\footnotetext{
${ }^{1}$ In [4] and [14] we consider the case where one agent has increasing marginal values and the other agent has decreasing marginal vaules.

${ }^{2}$ Here we assume ties go to agent 2 ; this is not essential, any other tie breaking rule can be used.
}

round is the terminal round. In fact, if there are no restrictions on the beliefs that can be attached with each agent, virtually any bidding strategy could be rationalized.

A bidding strategy is sophisticated if it maximizes an agent's payoff over final or expected final outcomes. The ability to make inferences on the final outcome requires the agent be sufficiently informed about the preferences and strategies of the other agent. We focus on sophisticated bidding in a setting of full information, but a similar analysis could be made for the case where the agent is Bayesian and has information about the distribution of the other agent's marginal values.

In the full information case each agent knows the number of units being sold, the bidding history of the other agent (and, of course, his own bidding history), and the valuations of the other agent. In particular both agents know when the last unit is being sold. The last round of the auction is identical to a standard second price auction for the $n$th good (the value for this good will of course depend on the outcomes of the previous rounds). Hence it is a dominant strategy for both agents to bid the marginal value on the last round. Since the marginal values are common knowledge, both agents know beforehand what are the allocation and payments on the last round and therefore their terminal payoffs. Thus, we can think of the penultimate round as an auction over the right to participate in one of two auctions in the last round. Since the payoffs of each one of these auctions is common knowledge, we can think of the penultimate round as a second price auction with valuations equal to these payoffs. It is therefore a dominant strategy in the penultimate round to bid the payoff from participating in one of the two possibilities of the last round.

We proceed in this way inductively until we reach the root. This shows that sophisticated bidding is the only strategy that survives iterative elimination of weakly dominated strategies. This does not rule out other equilibria and in fact there exist other Nash equilibria with higher payoffs for both agents (if for instance they conspire against the seller). However, these equilibria must rely on unreliable threats and commitments. We eliminate these equilibria from consideration by focusing on subgame perfect equilibrium. This discussion is summarized in the following theorem.

Theorem 1: In a sequential auction with fully informed agents, sophisticated bidding is the unique subgame perfect equilibrium after iterative elimination of weakly dominated strategies.

We define the equilibrium path to be the outcome produced when both agents use a sophisticated bidding strategy and the sequential allocation to be the allocation at the terminal node of the equilibrium path.

\section{B. Example}

Consider an example with $n=3$ goods. Suppose that agent 1 values each unit at $\$ 6$. Agent 2 values the first unit at $\$ 4.1$, the second at $\$ 3.1$ and the third unit at zero. Thus, the agents have the following marginal values: $u_{1}^{1}=u_{2}^{1}=u_{3}^{1}=6$, 


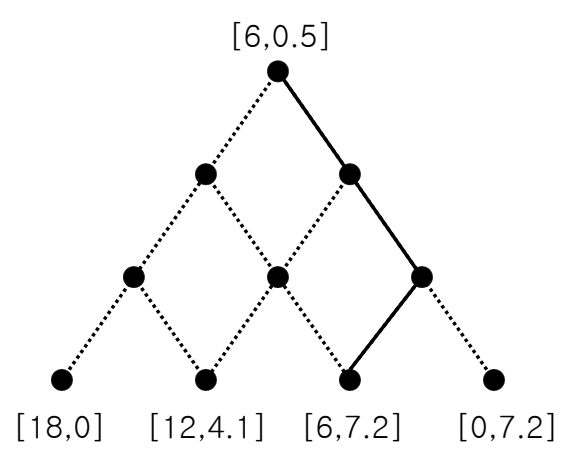

Fig. 1. An example graph $G$ for a sequential auction with $n=3$ goods. The final nodes are labeled with the utility of the corresponding allocation, assuming that the marginals are $u_{1}^{1}=u_{2}^{1}=u_{3}^{1}=6, u_{1}^{2}=4.1, u_{2}^{2}=3.1$ and $u_{3}^{2}=0$. The solid line shows the equilibrium path of the sequential second price auction. The final payoffs for both agents along this path are $[6,0.5]$.

$u_{1}^{2}=4.1, u_{2}^{2}=3.1$ and $u_{3}^{2}=0$. Figure 1 shows the graph $G$ corresponding to this example. Here, branches to the left (right) represent the case that agent 1 (2) wins a given round. The nodes at the bottom of the graph are labeled with the utility received by each agent at that outcome. For example, the final allocation $(s, t)=(3,0)$ is given by the leftmost final node on the graph. At this allocation, the total utility for agent 1 is 18 and that for agent 2 is 0 . Since Agent 1 values each unit more than agent 2, this is the efficient allocation. However, as we will show next, this is not the sequential allocation.

Let us examine sophisticated bidding for this example. Agent 1 should assume that agent 2 will bid 4.1 in every round until she is allocated at least one unit. Therefore, agent 1's payment for receiving all 3 goods will be $4.1 \times 3=12.3$ giving a payoff of $18-12.3=5.7$. On the other hand, if agent 1 does not bid on the first unit, she knows agent 2 will bid 3.1 on the remaining two units. Therefore, by losing the first round she can receive two units for a total payment of 6.2 resulting in a total payoff of 5.8. If she takes this reasoning even further agent 1 can abstain from obtaining the first two units. In this case agent 2's marginal value for the last unit drops to 0 and agent 1 gets it for free with total payoff 6 . Thus, in this setting agent 1's payoff is maximized when she is allocated only one unit. It can be shown using backward induction that this is indeed the equilibrium path for this example. This path is shown in bold in Fig. 1. The total utility of the sequential allocation $(1,2)$ is $6+7.2=13.2$, which is less than the total utility of 18 obtained at the efficient outcome. Therefore, the efficiency of the sequential auction in this example is $13.2 / 18=0.73$. In the following section we turn to bounding this loss in efficiency.

\section{A Bound ON THE EFFICIENCY}

For any decreasing marginal values $\left\{u_{i}^{1}\right\}_{i=1}^{n}$ and $\left\{u_{i}^{2}\right\}_{i=1}^{n}$, let $(k, n-k)$ denote the efficient allocation, and let $\left(k^{\prime}, n-k^{\prime}\right)$ denote the sequential allocation ( $k$ and $k^{\prime}$ are functions of the marginal valuations). The worst-case efficiency $\eta(n)$ for given $n$ is defined by ${ }^{3}$

$$
\eta(n)=\min _{\left\{u_{i}^{1}\right\},\left\{u_{i}^{2}\right\}} \frac{\sum_{i=1}^{k^{\prime}} u_{i}^{1}+\sum_{i=1}^{n-k^{\prime}} u_{i}^{2}}{\sum_{i=1}^{k} u_{i}^{1}+\sum_{i=1}^{n-k} u_{i}^{2}} .
$$

We refer to $1-\eta(n)$ as the worst-case efficiency loss. Our main result is the following.

Theorem 2: When both agents have decreasing marginal values, then for all $n$,

$$
\eta(n) \geq 1-e^{-1} .
$$

In other words, the worst-case efficiency is bounded by $1-$ $e^{-1}$ for any $n$. Moreover, it can be shown that the worst-case efficiency is decreasing with $n$, i.e.,

$$
\eta(1) \geq \eta(2) \geq \cdots \geq \eta(n) \geq \cdots \geq 1-\frac{1}{e},
$$

and the bound of $1-e^{-1}$ is asymptotically tight as $n \rightarrow \infty$.

The proof relies on the intuition that the worst-case efficiency in the equilibrium outcome occurs in the case when the utilities have the following form.

Definition 1: Agent 1's utilities are dominant if $u_{1}^{1} \geq \ldots \geq$ $u_{n}^{1} \geq u_{1}^{2} \geq \ldots \geq u_{n}^{2}$. We will also refer to this as a dominant utility profile. Agent 1's utilities are flat dominant if $u_{1}^{1}=$ $\ldots=u_{n}^{1} \geq u_{1}^{2} \geq \ldots \geq u_{n}^{2}$.

The efficient allocation for a dominant utility profile is to assign all units to agent 1 . In the sequential auction, however, agent 2 may receive one or more units, and this induces the efficiency loss. In fact there are examples where agent 2 may receive up to $n-1$ units.

\section{A. Equilibrium Path Payments}

We begin the proof of theorem 2 by determining the payment that agent 1 makes on each unit she receives in the sequential allocation.

Lemma 3: Suppose that agent 1 has a dominant utility profile. If $(s, t)$ is the sequential allocation, then $s \geq 1$ and agent 1 pays $u_{n-s+1}^{2}$ for each unit she receives.

Proof: We prove Lemma 3 by induction on the number of units allocated. It is immediate for $n=1$ since the auction is then a standard second price auction. For $n>1$ we can think of the root as a decision node between two alternatives:

1) Agent 1 receives a unit and both agents participate in an auction for $n-1$ units.

2) Agent 2 receives a unit and both agents participate in an auction for $n-1$ units.

If the equilibrium path allocates the first unit to agent 2 , then agent 1 pays nothing and the lemma follows by induction. This is because the equilibrium path for the $n$-unit auction contains the equilibrium path for the $(n-1)$-unit auction (subgame) rooted at node $(0,1)$, and the utilities associated with the sugbame have a dominant profile (i.e., $u_{1}^{1} \geq \ldots \geq$ $\left.u_{n-1}^{1} \geq u_{2}^{2} \geq \ldots \geq u_{n}^{2}\right)$.

\footnotetext{
${ }^{3}$ The quantity $1 / \eta(n)$ is sometimes referred to as the price on anarchy, see e.g. [13].
} 


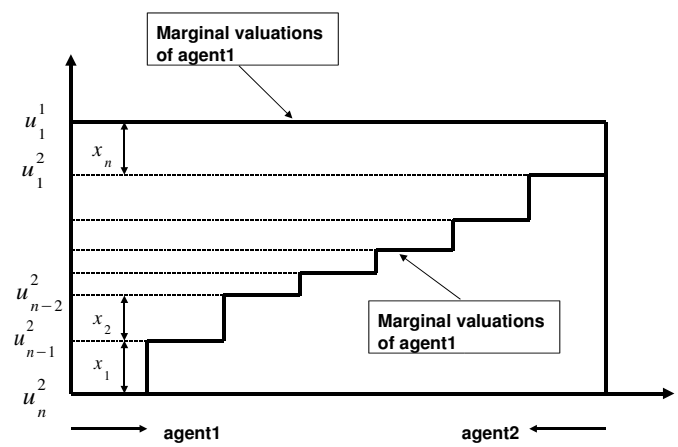

Fig. 2. The extremal case: Agent 1 has a constant marginal valuations and all the marginals of agent 2 are below the marginals of agent 1 . The axis for agent 1 is from left to right and the axis for agent 2 is from right to left.

If the first unit is allocated to agent 1 , it suffices to show that she pays $u_{n-s+1}^{2}$. Agent 2 bids the difference between participating in an $(n-1)$-unit sequential auction and receiving an extra unit, and participating in the same auction without the extra unit. This difference is therefore the value of the extra unit, $u_{n-s+1}^{2}$, which is agent 1 's payment.

Under the assumptions of Lemma 3 , we can write the payoff of agent 1 for any terminal allocation $(s, t)$, assuming that this is the sequential allocation. Furthermore, since agent 1 's utility is dominate, she can essentially choose the terminal allocation, which gives her the highest pay-off. Her choice will be the sequential allocation. This is summarized in the following corollary.

Corollary 4: Suppose agent 1 has a dominant utility profile. Then, the allocation $(s, t)$ is the sequential allocation if and only if

$$
\sum_{i=1}^{s}\left(u_{i}^{1}-u_{n-s+1}^{2}\right) \geq \sum_{l=1}^{r}\left(u_{l}^{1}-u_{n-r+1}^{2}\right),
$$

for any $r \in\{1, \ldots, n\}$.

\section{B. Bounds for Flat Dominant Valuations}

Next we assume a flat dominant utility profile (i.e., $u_{1}^{1}=$ $\ldots=u_{n}^{1} \geq u_{1}^{2} \geq \ldots \geq u_{n}^{2}$ ). If we let $x_{i}=u_{n-i}^{2}-u_{n-i+1}^{2}$ for $i=1, \ldots, n-1$, and $x_{n}=u_{1}^{1}-u_{1}^{2}$ (see Fig. 2), equation (7) can be written as

$$
s \cdot \sum_{k=s}^{n} x_{k} \geq r \cdot \sum_{k=r}^{n} x_{k}
$$

for any $r \in\{1, \ldots, n\}$.

The difference in value between the efficient allocation and the sequential allocation $(s, t)$ is

$$
n \cdot u_{1}^{1}-\left(s \cdot u_{1}^{1}+\sum_{k=1}^{n-s} u_{k}^{2}\right)=\sum_{k=s+1}^{n}(k-s) \cdot x_{k} .
$$

Likewise, we have $\sum_{k=1}^{n} x_{k}=u_{1}^{1}-u_{n}^{2}$, and so the efficiency loss can be written as

$$
\frac{\sum_{k=s+1}^{n}(k-s) \cdot x_{k}}{n \cdot\left(\sum_{k=1}^{n} x_{k}+u_{n}^{2}\right)} \text {. }
$$

The next lemma bounds this for a given allocation of $j$ units to agent 1 .

Lemma 5: The maximum efficiency loss for the sequential allocation $(j, n-j)$ assuming a flat dominant utility profile is $\frac{j}{n} \sum_{k=j}^{n-1} \frac{1}{k+1}$.

Proof: Let $x_{1}, \ldots, x_{n}$ be the solution to the following linear program:

$$
\begin{array}{rc}
\max _{\left\{x_{i}\right\}} & \phi\left(x_{1}, \ldots, x_{n}\right):=\sum_{k=j+1}^{n}(k-j) \cdot x_{k}, \\
\text { subject to: } & j \cdot \sum_{k=j}^{n} x_{k} \geq r \cdot \sum_{k=r}^{n} x_{k}, \quad \forall r \neq j, \\
\sum_{k=1}^{n} x_{k}=u_{1}^{1}-u_{n}^{2}, \\
x_{1}, \ldots, x_{n} \geq 0 .
\end{array}
$$

From the discussion preceding Lemma 5, the maximum efficiency loss for the sequential allocation $(j, n-j)$, assuming a flat dominant utility profile, is

$$
\max _{u_{1}^{1}, u_{n}^{2}: u_{1}^{1}>u_{n}^{2} \geq 0} \frac{\phi\left(x_{1}, \ldots, x_{n}\right)}{n\left(\sum_{k=1}^{n} x_{k}+u_{n}^{2}\right)} .
$$

First note that the linear program only depends on $u_{1}^{1}-u_{n}^{2}$, and (15) is decreasing in $u_{n}^{2}$. Hence we can always increase the efficiency loss by setting $u_{n}^{2}=0$. In addition, because the objective function only depends on $x_{j+1}, \cdots, x_{n}$, we set $x_{1}=\cdots=x_{j-1}=0$ to make the largest feasible region. With this choice of $x_{i}$, the only constraints in (12), which can be binding, are those for $r>j$. It is easy to see that at optimality, the remaining constraints are binding. Therefore, the constraints (12) and (13) can be written as the following linear system:

$$
\begin{aligned}
x_{j}+x_{j+1}+x_{j+2}+\ldots+x_{n} & =u_{1}^{1} \\
x_{j+1}+x_{j+2}+\ldots+x_{n} & =\frac{j}{j+1} u_{1}^{1} \\
x_{j+2}+\ldots+x_{n} & =\frac{j}{j+2} u_{1}^{1} \\
& \vdots \\
x_{n} & =\frac{j}{n} u_{1}^{1} .
\end{aligned}
$$

This set of equations gives the following unique feasible solution.

$$
x_{k}= \begin{cases}\frac{j}{k(k+1)} & k=j, \ldots, n-1, \\ \frac{j}{n} & k=n .\end{cases}
$$

Hence from (15), the maximum efficiency loss is $\frac{j}{n} \sum_{k=j}^{n-1} \frac{1}{k+1}$.

From Lemma 5, it follows that the worst-case efficiency of the sequential auction given that agent 1 has a flat dominant utility profile is

$$
\eta^{\prime}(n)=\min _{j \in[1, \ldots, n]}\left\{1-\frac{j}{n} \sum_{k=j}^{n-1} \frac{1}{k+1}\right\} .
$$

As $n \rightarrow \infty$, it can be easily shown that $\eta^{\prime}(n) \rightarrow 1-e^{-1}$. 


\section{Proof of Theorem 2}

We next show that the flat dominant utility profile achieves the worst-case efficiency, namely, $\eta^{\prime}(n)=\eta(n)$. This completes the proof of Theorem 2. We do so in two steps. First we show that for any pair of marginals for agents 1 and 2 there exists a pair of marginals where agent 1's marginals are dominant over those of agent 2 with a lower efficiency in the outcome. Then we show that changing a dominant utility profile to a flat dominant profile can only decrease efficiency.

Step 1: For any pair of decreasing marginal values $u_{1}^{1} \geq$ $\ldots \geq u_{n}^{1}$ and $u_{1}^{2} \geq \ldots \geq u_{n}^{2}$, let $k$ and $n-k$ be the efficient allocation for agents 1 and 2. After auctioning $l(\leq n)$ units among $n$ units, the sequential game reaches a decision node where either agent 1 or agent 2 obtains his efficient allocation ( $k$ for agent 1 or $n-k$ for agent 2 ). Since one agent meets his efficient allocation after $l$ units are assigned, the marginal values of the remaining units for this agent must be smaller than that for the other agent. (See Fig. 3). Up to this decision node, there is no loss in efficiency. Any efficiency loss in the final allocation procures in the subgame tree rooted at this decision node. Therefore, efficiency loss of the full game tree cannot be larger than the efficiency loss of this subgame tree. This shows that for any pair of marginal values $u_{1}^{1} \geq, \ldots, \geq$ $u_{n}^{1}$ and $u_{1}^{2} \geq, \ldots, \geq u_{n}^{2}$, there exists a pair $\bar{u}_{1}^{1}, \ldots, \bar{u}_{n}^{1}$ and $\bar{u}_{1}^{2}, \ldots, \bar{u}_{n}^{2}$ satisfying $\bar{u}_{1}^{1} \geq \ldots \geq \bar{u}_{n}^{1} \geq \bar{u}_{1}^{2} \geq \ldots \geq \bar{u}_{n}^{2}$ with a lower efficiency in the outcome of the sequential auction. The worst-case efficiency must always correspond to a dominant utility profile.

Step 2: Suppose that agent 1 has a dominant utility profile (i.e., $u_{1}^{1} \geq \ldots \geq u_{n}^{1} \geq u_{1}^{2} \geq \ldots \geq u_{n}^{2}$ ). From Corollary 4 , if $(s, t)$ is the sequential allocation, then

$$
\sum_{i=1}^{s}\left(u_{i}^{1}-u_{n-s+1}^{2}\right) \geq \sum_{l=1}^{r}\left(u_{l}^{1}-u_{n-r+1}^{2}\right)
$$

for any $r \neq s$. If we replace the marginals of agent 1 with the following flat dominant marginals $\bar{u}_{1}^{1}=\ldots=\bar{u}_{n}^{1}=u_{n}^{1}$, then

$$
s \cdot\left(\bar{u}_{1}^{1}-u_{n-s+1}^{2}\right) \geq r \cdot\left(\bar{u}_{1}^{1}-u_{n-r+1}^{2}\right) .
$$

This implies that for the marginals $\bar{u}_{1}^{1}=\ldots=\bar{u}_{n}^{1} \geq u_{1}^{2} \geq$ $\ldots \geq u_{n}^{2}$, the sequential equilibrium $(\bar{s}, \bar{t})$ satisfies $\bar{s} \leq s$. In other words, 'flattening' the dominant utilities can only decrease efficiency.

\section{Constructive Examples for the Bound}

Next we construct examples to show that the bound of Theorem 2 is asymptotically tight. First consider the sequential auction for $n=2$ goods. If $u_{1}^{1}=u_{2}^{1}=1$ and $u_{1}^{2}=\frac{1}{2}+\varepsilon_{1}$, $u_{2}^{2}=0$, then it can be shown that the sequential allocation is $(1,1)$. Instead of getting two goods with payoff $1-2 \varepsilon_{1}$, agent 1 is better off by just getting one good with payoff 1. The efficiency of this auction with the marginal values above approaches $\frac{1+\frac{1}{2}}{1+1}=\frac{3}{4}$ as $\varepsilon_{1} \rightarrow 0$. It can be shown that this is the worst-case efficiency among all possible profiles of marginal values for two agents and two goods. Therefore, $\eta(2)=\frac{3}{4}$.

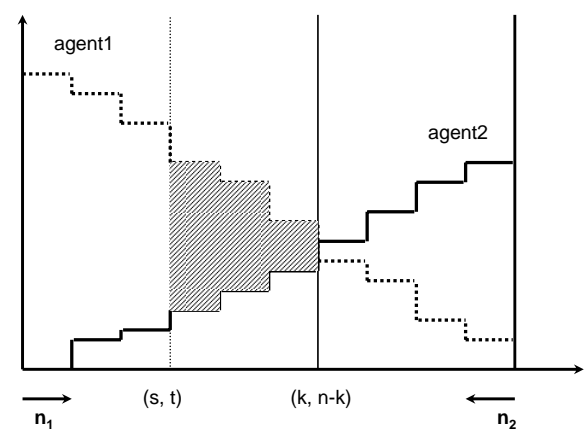

Fig. 3. Marginal valuations of two agents. $n_{1}\left(n_{2}\right)$ is the number of goods that agent 1 (2) obtains along the sequential auction. $(k, n-k)$ is the optimal allocation and $(s, t)$ is the sequential allocation. The shadowed region shows the efficiency loss.

Similarly, we can construct the marginal values of each agent to get the worst-case efficiency of the sequential auction for all $n>2$. Consider the following profile of marginal values of $n$ goods: $u_{1}^{1}=\ldots=u_{n}^{1}=1$ for agent 1 and $u_{1}^{2}=1-\frac{j}{n}+\varepsilon_{1}, u_{2}^{2}=1-\frac{j}{n-1}+\varepsilon_{2}, u_{3}^{2}=1-\frac{j}{n-2}+$ $\varepsilon_{3} \ldots, u_{n-j}^{2}=1-\frac{j}{j+1}+\varepsilon_{n-j}, u_{n-j+1}^{2}=0, \ldots, u_{n}^{2}=0$ for agent 2 , where $j \in\{1, \ldots, n\}$. The sequential auction ends with the allocation $(j, n-j)$ according to Corollary 4 . If agent 1 receives $j$ goods, her payoff after the auction ends is $j \cdot 1$. However, if agent 1 receives $j-1$ goods, her payoff becomes $(j-1) \cdot\left(1-u_{n-j}^{2}\right)=j-(j+1) \cdot \varepsilon_{n-j}$, which is smaller than $j$. In a similar way, agent 1's payoff is smaller than $j$ if she is allocated $r \neq j$ goods. Therefore, the sequential allocation is $(j, n-j)$. As the $\varepsilon_{k}$ 's approach zero, the efficiency of this outcome approaches

$$
\begin{aligned}
\frac{j+\sum_{k=1}^{j} u_{k}^{2}}{n} & =\frac{j}{n}+\sum_{k=1}^{n-j} \frac{1}{n} \cdot\left(1-\frac{j}{n-k+1}\right) \\
& =1-\frac{j}{n} \sum_{k=j}^{n-1} \frac{1}{k+1} .
\end{aligned}
$$

Minimizing (20) over $j \in[1, \ldots, n]$ gives the worst-case efficiency for this class of valuations, i.e.,

$$
j^{*}=\arg \min _{1 \leq j \leq n}\left\{1-\frac{j}{n} \sum_{k=j}^{n-1} \frac{1}{k+1}\right\} .
$$

Note that this is the same as the expression in (17) and hence it follows that these allocations give the worst-case efficiency for each $n$.

Table I shows the marginal values of two agents that give the lowest efficiency $\eta(n)$, which is also shown. As can be seen, $\eta(n)$ is decreasing with in $n$, i.e.,

$$
\eta(1) \geq \eta(2) \geq \cdots \geq \eta(n) \geq \cdots \geq 1-\frac{1}{e} .
$$

As $n \rightarrow \infty$, these quantities approach the bound asymptotically. 


\begin{tabular}{|c|c|c|c|}
\hline $\mathrm{n}$ & Marginals & $j^{*}$ & $\eta(n)$ \\
\hline 2 & 1,$1 ; 1 / 2+\varepsilon_{1}, 0$ & 1 & $3 / 4$ \\
\hline 3 & $1,1,1 ; 2 / 3+\varepsilon_{1}, 1 / 2+\varepsilon_{2}, 0$ & 1 & $13 / 18$ \\
\hline 4 & $1,1,1,1 ; 1 / 2+\varepsilon_{1} ; 1 / 3+\varepsilon_{2}, 0,0$ & 2 & $17 / 24$ \\
\hline$\vdots$ & $\vdots$ & $\vdots$ & $\vdots$ \\
\hline$\infty$ & & & $1-\frac{1}{e}$ \\
\hline
\end{tabular}

TABLE I

MARGINAL VALUES AND CORRESPONDING WORST-CASE EFFICIENCY ACHIEVED BY THE SEQUENTIAL AUCTION FOR GIVEN $n$.

\section{CONCLUSION}

We have considered a sequential second price auction of $n$ homogeneous goods for two agents with decreasing marginal values. This was motivated by a dynamic spectrum sharing application, where the goods could represent units of bandwidth. Our results show that the worst-case efficiency of subgame perfect equilibrium is non-increasing function in total number of goods $n$ and is bounded by $1-e^{-1}$. For the spectrum sharing application, this suggests that that a potential direction is to study how finely to partition the available bandwidth if it is to be sequentially auctioned. Our results are preliminary; in particular we have only considered two agents ${ }^{4}$ and assumed full information. Relaxing either of these assumptions are potential directions for future work.

\section{REFERENCES}

[1] A. A. Lazar and N. Semret, "Design and analysis of the progressive second price auction for network bandwidth sharing," Telecommunication Systems - Special Issue on Network Economics, 1999.

[2] R. Johari and J. N. Tsitsiklis, "Efficiency loss in a network resource allocation game," Mathematics of Operations Research, vol. 29, no. 3, pp. 407-435, August 2004.

[3] J. Huang, R. Berry, and M. L. Honig, "Auction-based spectrum sharing," ACM/Springer Mobile Networks and Applications Journal (MONET), vol. 11, pp. 405-418, 2006.

[4] J. Bae, E. Beigman, R. Berry, M. L. Honig, and R. Vohra, "Efficiency of sequential bandwidth and power auctions with rate utilities," in IEEE 2nd International Conference on Congnitive Radio Oriented Wireless Networks and Communications (CrownCom), Orlando, FL, 2007.

[5] "Order and further notice of proposed rule making on smart radios," Federal Communications Commission Report 03-322, 2003.

[6] "The development of secondary markets - report and order and further notice of proposed rule making," Federal Communications Commission Report 03-113, 2003.

[7] C. Boutilier, M. Goldszmidts, and B. Sabata, "Sequential auctions for the allocation of resources with complementarities," in Proceedings of 16th International Joint Conference on Artificial Intelligence (IJCAI-99), 1999, pp. 527-534.

[8] C. Mezzetti, A. Pekec, and I. Tsetlin, "Sequential vs. single-round uniform auctions," December 2004

[9] P. R. Milgrom and R. J. Weber, "A theory of auctions and competitive bidding," Econometrica, vol. 50, no. 5, pp. 1089-1122, September 1982.

[10] R. J. Weber, "Multiple-object auctions," 1983, discussion Papers 496, Northwestern University, Center for Mathematical Studies in Economics and Management Science.

[11] J. T. yi Wang, "The ebay market as sequential second price auctions: Theory and experiments," November 2006, unpulbished manuscript.

[12] K. Steiglitz, Snipers, Shills, and Sharks: eBay and Human Behavior. Princeton University Press, March 2007.

[13] T. Roughgarden and E. Tardos, "How bad is selfish routing?" Journal of the ACM, vol. 49, no. 2, pp. 236-259, March 2002.

${ }^{4}$ In [14] we consider more than two agents with decreasing marginal values.
[14] J. Bae, R. B. Eyal Beigman, M. L. Honig, and R. Vohra, "Sequential bandwidth and power auctions for distributed spectrum sharing," submitted to IEEE Journal on Selected Areas in Communications. 